\title{
Future smart grid communication-deployment of IoT: opportunities and challenges
}

\author{
Payal Soni, J. Subhashini \\ Department of Electronics and Communication, SRMIST, SRM University, Kattankulathur, Kancheepuram Dt., \\ Tamilnadu, India
}

\begin{tabular}{|c|c|}
\hline Article Info & ABSTRACT \\
\hline Article history: & India's electrical power system grid also known as the power grid is serving \\
\hline Received Aug 9, 2020 & $\begin{array}{l}\text { us from a very long time. In this duration, there were no major developments } \\
\text { or changes reported in the power grid system. Electrical power consumer }\end{array}$ \\
\hline Revised Apr 23, 2021 & demand is increasing drastically and the present grid system is not able to \\
\hline Accepted Apr 30, 2021 & $\begin{array}{l}\text { fulfil these emerging requirements. To fulfil the requirements of future power } \\
\text { load, we need a modified system which has to be reliable, secure, intelligent }\end{array}$ \\
\hline Keywords: & $\begin{array}{l}\text { and efficient. By converting the power grid into the smart grid will be a } \\
\text { promising solution for adopting the above properties. Communication }\end{array}$ \\
\hline Communication technology & \\
\hline IoT & $\begin{array}{l}\text { expenditure on electricity demand by using smart home appliance, to keep } \\
\text { away from the rush hours and also make use of the renewable energy instead }\end{array}$ \\
\hline Smart grid & from utility, is a great example of deployment of internet of things (IoT) in \\
\hline Smart meter & $\begin{array}{l}\text { grid communication. In this paper, we have provided a survey of different } \\
\text { communication technology, applications, benefits and challenges in } \\
\text { communication infrastructure, spatially IoT. }\end{array}$ \\
\hline
\end{tabular}

This is an open access article under the CC BY-SA license.

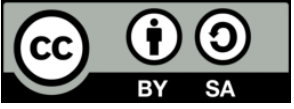

Corresponding Author:

Payal Soni

Department of Electronics and Communication Engineering

SRMIST, SRM University

Kattankulathur, Kancheepuram Dt., Tamilnadu, India

Email: payals@srmist.edu.in

\section{INTRODUCTION}

Smart grid is an electrical grid with automation, communication and knowledge systems that can monitor power flows from generation to consumption and control the power flow to match generation in real time [1]. Due to change in global conditions and growing populations over the past decades, there is increase in clean energy demand on global basis. The rising electricity demand means the nonlinearity in power transmission and distribution network, and congestion, outage, fluctuation in voltage and frequency are the measure issues that becomes cause of blackouts [2]. Smart grid has self healing property equipments that have real time data to decrease system outage and losses, voltage level fluctuations etc [3]. Moreover, the global integration of renewable energy sources with main electricity generation system has great initiatives in this century, such as integration, stabilizing system voltage, and increment in energy storage devices, these issues also come under challenges [4]. The key aspect of the smart grid is more reliable and quick data information for efficient delivery of power from the Generation to distribution. The communication infrastructure is an important part of smart grid. By using communication technologies, all the necessary information about power generation to consumption and from consumer to generation station could be collected and analyzed for smart operation of grid [5]. Information such as amount of power transmission, distribution losses and consumption and fault location etc could be analyzed by bidirectional communication 
between fields connected smart meters, which is called as advanced metering infrastructure (AMI). AMI is a key component of smart grid [6]. To control and measure the abnormal parameters value, self healing, transfer reliable bidirectional information between smart meters and power consumption; deployment of internet of things is necessary [7]. Smart grid has numerous optimistic qualities such as:

- Real time monitoring and control and automatic fault management and quicker restoration.

- Improved energy management and control and in-house real time data consumption displays.

- Tracking and managing energy usage at consumers end and conserve electricity.

- Real time information facilitates by wireless and cellular technologies.

Today's electric-power-system has vertical structure, one-way information flow and centralized energy production from generation to distribution and centralizes system, whereas smart grid is a decentralise grid, bidirectional flow of information and has non vertical structure [8]. Past grid monitoring, diagnostic and controlling systems are usually realized through wired communications [9]. However, the wired monitoring systems require expensive communication cables to be installed; therefore, wireless communication plays an important role in monitoring and diagnostic to get better system reliability and effectiveness by optimizing the management of electric power systems with price effective aspects [10]. Smart grid has active customer participation; it has automatically detected the problem and responds faster means generally focus on prevention of fault [11].

Smart grid is a modern electric power system infrastructure for enhancing efficiency and reliability through the system using automatic metering infrastructure that has automated control for high-power converters, modern communications infrastructure, sensing and metering technologies, and modern energy management techniques [12]. The recent communication abilities of the active grid are limited to small-level system monitoring and control, such as power-line communications [13]. It has sluggish response to power quality issues, modest incorporation of processing data through quality supervision. Smart grid system helps to reducing the transmission and distribution losses using real time monitoring and control capabilities of communication technologies to transfer electricity in a more effective and reliable way [14]. The system state evaluation and control are important concern to make sure that about smart grid stability and reliability [15]. A Comparison is based upon diagram is shown below in Figure 1. Nature of smart grid over the past grid as: - The end user is involved and participative in the grid operations.

- Non vertical power distribution system with integration of renewable sources of energy.

- Focus on preventive maintenance of system and resilient to cyber attacks, rapid restoration abilities.

- Integrated algorithm for optimization and efficiency.

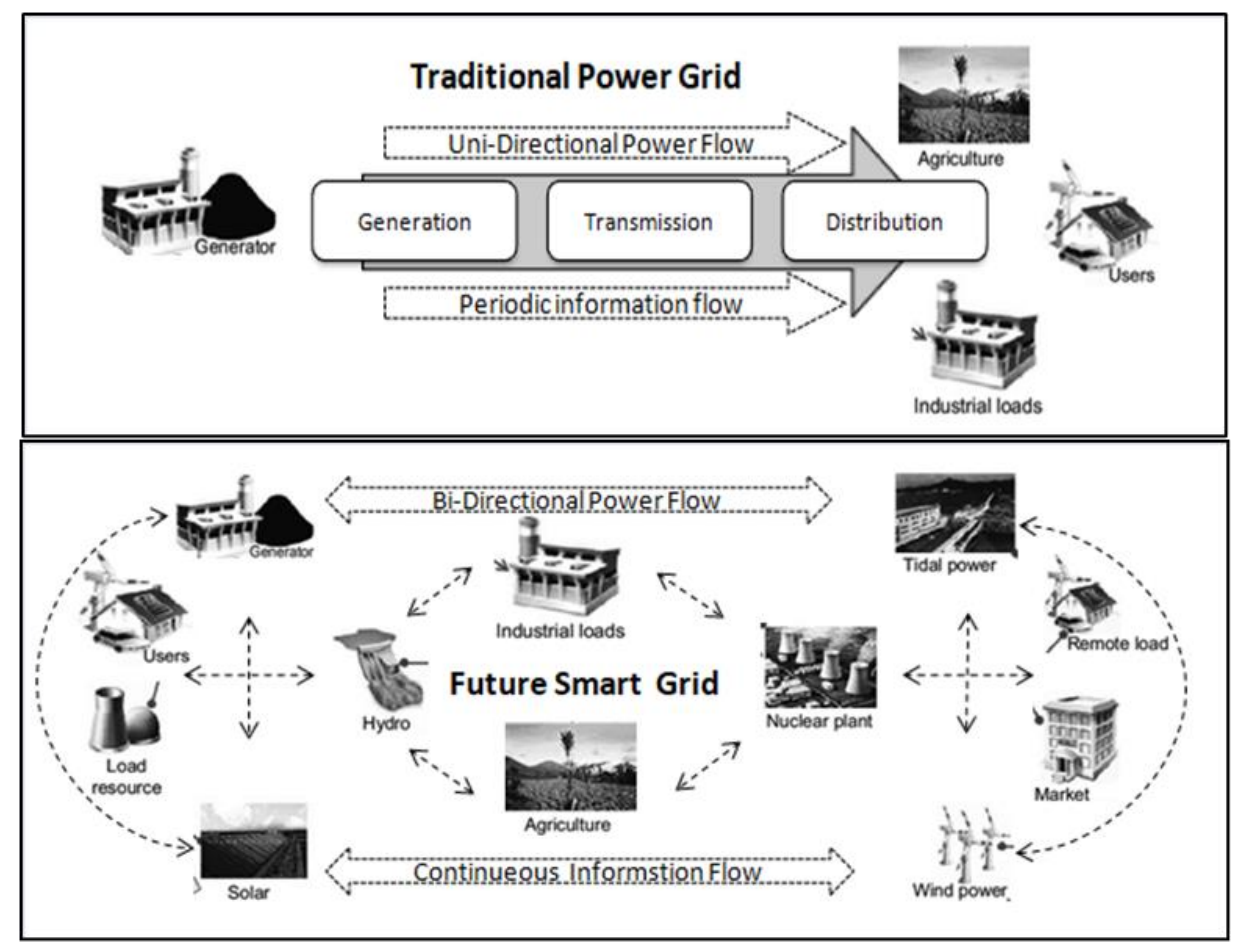

Figure 1. Traditional grid and future smart grid 


\section{COMPREHENSIVE THEORETICAL BASIS}

Different communication technology has different characteristics. To choose a particular communication technology for different power subsystem, a systematic study is necessary to complete the application requirements with the technical aspects [16]. This three-layered construction comprises a Wide area network (WAN), local area network (LAN) and personal area network (PAN) [17]. According to distance of field, speed of information exchange and collecting data with small delay, smart grid communication infrastructure in the smart grid system can be shown by a layered construction, as given in Figure 2.

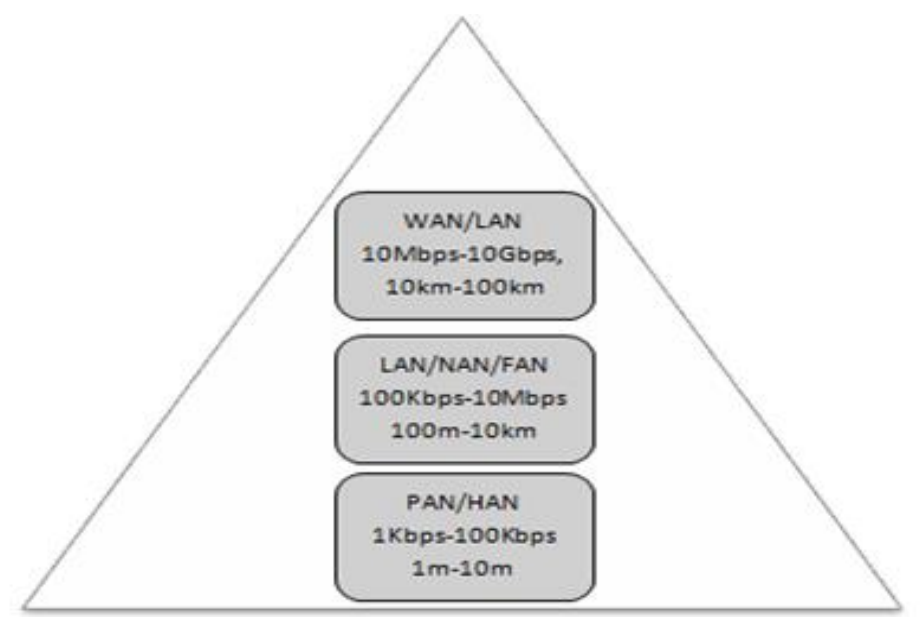

Figure 2. Specification required for communication network architecture in smart grid

\subsection{Wide area network}

WAN offers two-way communications for intelli grid generating substation control centre, transmission substation control centre to distribution substation control centre. All real time information at the substation is delivered by WANs using SCADA, RTUs, smart meters and sensors which are connected to grid [18]. WAN can be implemented using a variety of communication technologies, these are fibre, Power line communication (PLC) etc. Optical fibre also used for data exchanging between subsystem substations high transmission capacity and small delay [19].

\subsection{Local area network and neighbourhood area network/field area network}

According to the distance between distribution grid, LAN is also used to transfer real time information. It supports real time energy consumption data from customers in a neighbourhood to a utility [20]. Various communication technologies come under this network, for instance ZigBee, WLAN, PLC and several extensive-distance communication technologies [21].

\subsection{Personal area network}

Home area network and building area network comes under the personal area network, they provide wireless communication to sharing information amongst all customer on the utility side [22]. PAN provides real time information from the end-user side. A personal area networks are containing selection of IoT devices, such as a home entrance, smart meters, sensor, smart home appliances for home automation and electric vehicles [23]. For a Reliable operation in smart grid from generation to consumer end, real-time data transfer by WAN/MLAN/HAN/PAN/NAN are analyse and control by smart grid, as shown in Figure 3.

\subsection{Overview of different types of communication technology}

A communication technology plays an important role in smart grid communication infrastructure. It is heart of smart grid, without two-way communication, smartness of the system not possible. Future smart grids will be more intelligent, more autonomous and better integrate demand-side DERs [24]. Communications technologies supported by both communications, employed in real information communication connecting all electrical appliances placed in grid such as smart meters, sensors, PMUs etc. The main advantages of wireless technologies over wired technologies, such as low-cost, use fewer infrastructures, easiness in connection, mobility, high data speed, less magnetic effect [25]. However, some 
disadvantages like noise interference, nature of transmission path causes attenuation, the life of the battery. Conversely, wired communication has less interference problems and it's free from particular frequency band [26]. Because of same unlicensed ISMB and interference occurs amongst other $2.4 \mathrm{GHz}$ wireless networks, such as WLAN, ZigBee based wireless technologies. The difference between communication technologies has been shown in Table 1 [27].

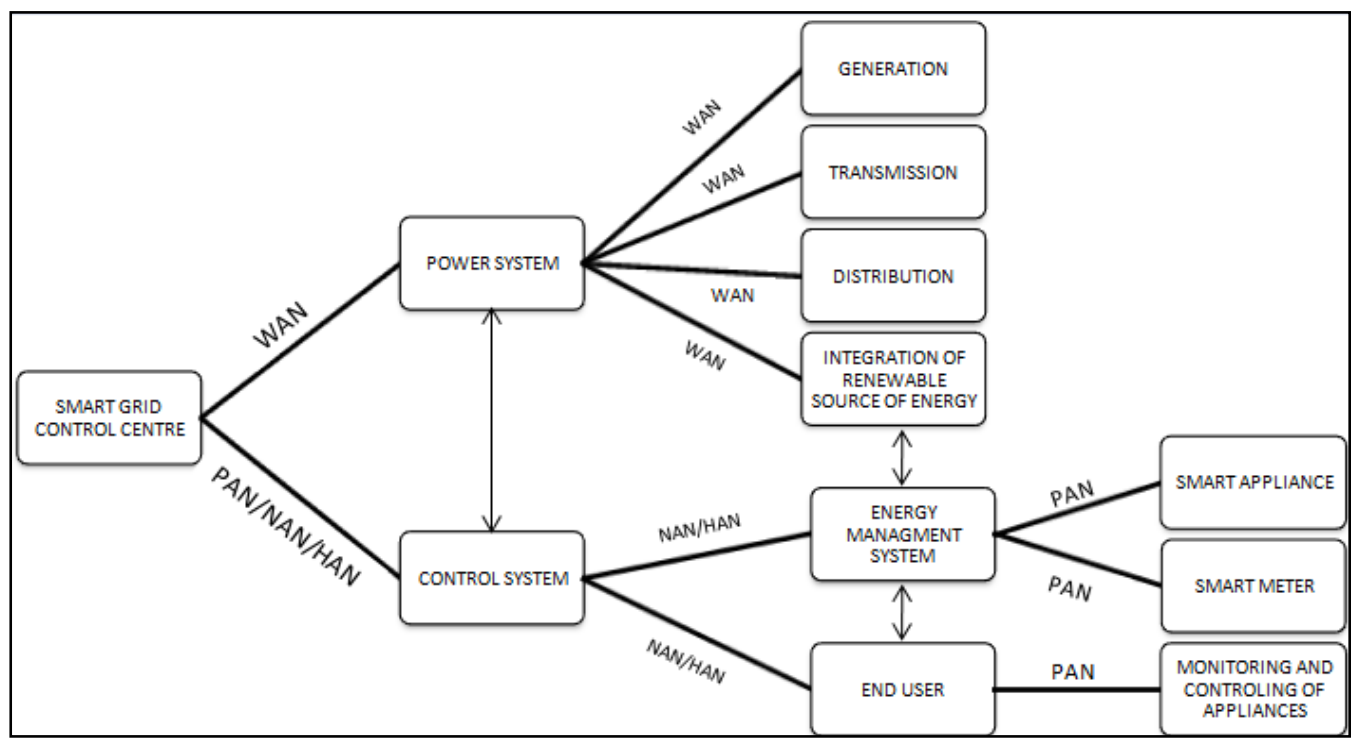

Figure 3. Proposed communication network architecture

Table 1. Comparison of wired and wireless communication technology

\begin{tabular}{lcc}
\hline Parameter & Wired Communication & Wireless Communication \\
\hline Speed of operation & Low & High \\
System bandwidth & Low & High \\
Cost & High & Low \\
Installation & Difficult & Easy \\
Mobility & Zero & Flexible \\
Network coverage extension & Cover a particular distance & Cover large distance \\
Channel Interference & No & Present \\
Reliability & High & High \\
QoS & Less & High \\
Speed of operation & Low & High \\
\hline
\end{tabular}

\section{DEPLOYMENT OF IOT IN SMART GRID}

To reduce the total load consumption during peak hours and improve the quality of services, deployment of IoT has several benefits for prosumers [28]:

- Decrement of transmission and distribution losses.

- Peak load, load shaving management.

- Deregulated market, Higher Renewable energy integration.

- Higher quality services provide by power companies at reduces cost.

- High grid vision for meeting demand response and self-healing qualities of grids.

The smart grid that is taken into account united of the foremost vital infrastructures is outlined because the electric power system increased with important information and control technologies and integration of renewable energy is seen united of the most important wireless (IoT network) network [29]. The smart grid can occupy outsized numbers of smart objects/things: smart meters, good appliances, sensors, actuators-cars, etc. Implementation in side today's grid be capable of improve every feature of the electrical Supply system, with generation, transmission, distribution and electric energy consumption, encourage customer to change patterns of electricity consumption according time and level of electric supply demand [30]. IoT technology is having wide range of applications. It means that the applications are specifics and the applications are dissimilar as of one device to other devices. There are essential points shared by devices 
[31]. As technical point of view all networks are realistic for real information collection, in real time, with low latency and effective in price [32].

\subsection{IoT at distribution side of smart grid}

Power distribution is the main subsystem of smart grid or power system, all consumers, for example industries, agriculture and housing societies get electricity from distribution substation according to their requirement, using IoT application all users can maintain the proper effective use of electricity and make consumption price effective. The main applications are:

\subsubsection{The requirement of IoT at generation side}

Monitoring of fuel quantity, integration of renewable energy source like wind, solar, biomass monitoring, monitoring of an environmental effect like carbon emission, energy storage monitoring, system monitoring etc.

\subsubsection{The requirement of IoT transmission side}

Electric transmission system monitoring, transmission tower protection, intellectual transmission substation, distribution system automation like SCADA system, EMS, condition monitoring, operation etc.

\subsubsection{The requirement of IoT at distribution side}

Smart meters, ICT Equipment, sensors, EV charging, efficiency monitoring, distribution side energy administration, smart room, to maintain less energy consumption in household appliances like washing machine, refrigerator, air conditioner, computer etc.

\subsection{IoT at consumer end of smart grid}

The Smart grid systems has real-time two-way communications that carry the inclusive power system management and control responsibilities which, requires real time and data-intensive information exchange [33]. Architecture layers of the smart grid at the distribution side shown in Table 2.

Table 2. Communication technologies and their applications

\begin{tabular}{lll}
\hline Name of Technology & Application & \\
\hline & Z-Wave & home automation; automatic meter reading \\
& Bluetooth Mesh & Home automation \\
& Li-If & Individual energy consumption application \\
& Wi-Fi & Distribution and substation automation \\
& ZigBee & Electrical Energy monitoring, home automation \\
& $5 \mathrm{G}$ & Monitoring and control in the distributed system \\
& Cellular Comm. & Monitoring and management of DERs; SCADA \\
& WiMAX & Real-time fault detection \\
& Wireless mesh & Substation Automation \\
& NB-IoT & Home Automation \\
& LoRa WAN & towers online power and fault monitoring \\
& Ethernet & Physical network infrastructure \\
PLC & Automatic meter reading \\
& Digital subscriber lines & Smart metering \\
\hline
\end{tabular}

\section{OPPORTUNITIES TO USE THE IOT IN SMART GRID}

In recent times, IoT has been extensively known as a promising technology for enhancement the system efficiency for above subsystems, to make power system smart [34]. Combination of advanced communication and information technologies makes electric power system smarter than past [35]. Essential opportunities for using IoT in distribution side smart grid are smart buildings, agriculture, medical and health care, institutions, transportation, energy management and environmental monitoring system [36]. This architecture is suitable for smart energy management and used for all costumers such as smart home, agriculture medical, and environmental parameters monitoring. Due to best features of AMI, self healing, real time information from smart sensors in the field, consumer can get best support at the time of any disturbance occurs related to electricity and reduce electricity bills to proper use of electricity, as shown in Figure 4. 


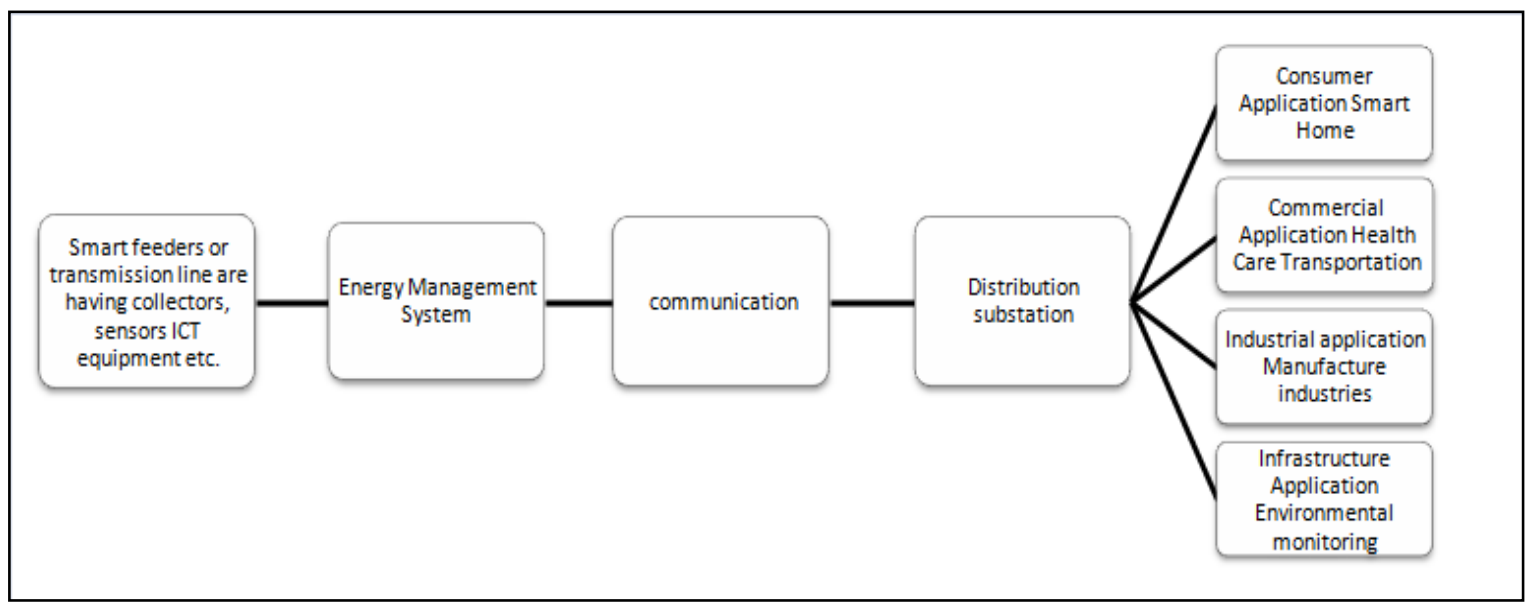

Figure 4. Architecture of smart grid, distribution side

\section{CHALLENGES}

The Internet of Things possibly a recent wireless communication concept, within which the user objects are capable for exchanging for information, using suitable protocol that makes them capable to transfer information's with each other and with other clients, turning into correlate degree integral a part of the internet. Smart grid has bidirectional power and communication to accomplish grid efficiency, reliability analysis, pricing, and security analysis and safety standards. However, these facilities, smart grids face network security challenges for example access data control, outage acceptance, network privacy etc [37]. A healthy communication infrastructure has great roll in smart grid, also improve the difficulties in real time fault finding, blackouts, voltage fluctuation, storage capacity etc [38], using communication technologies in smart grid, smart meters, PMUs and provides real time information about outage, energy consumption [39].

\subsection{Latency}

Delay of data transmission between smart meters or sensors such as smart metering systems and home energy management is an important parameter in Smart grid distribution system [40]. The important quality of smart grid is monitoring of system conditions, protection against fault occurrence, system automation, optimization, integration of renewable energy and protection of the power and information flow from utility to the consumers side equipments [41]. An Energy-conscious smart house is a suburban construction equipped with a Smart Metering system capable factual time measurement and control of the electricity consumption of appliances connected in smart house [42].

\subsection{High data rate}

There are large numbers of smart meters, sensors RTUs, PMUs are deployed through the smart grid system, a huge amount of real time information or data continues transferred by communication technologies [43]. Therefore high data rate communication is necessary for bidirectional flow of information in real time manner; it should be more concentrating on improving existing communication. It is a future requirement to make smart grid more efficient and realistic. In present days LPWAN Technologies such as NB-IoT, Sigfox, LoRA and Li-Fi is quite high as compare to other communication technologies but it must be more improved by adopting new techniques [44].

\subsection{Interference}

Interference in the communication channel is one of the main challenges [45]. Personal area networks are most probably installed in suburban area, HAN is used for a home automation application, it should have enhanced network security to make sure that essential and correct real time information is communicate to the smart meter [46]. HAN/LAN/FAN is directly connected to Smart meters, sensors, PMUs, due to same frequency band of different technologies interference occur between networks and causes unreal and unreliable transmission of signals from smart meters, to eliminate this problem, research on developing algorithms to decrease interference is one of the challenges [47]. In the future Smart home, dozens of devices will communicate with each other using communication [48]. 


\subsection{Security and privacy}

Network Security, also is an important challenge in deployment of IoT application in smart grid, to transmit data secure communication is required [49]. It can be hacked by other persons. Security hazards involved the exposing of confidential records, illegal entrance and insertion of wrong data in order to change the regular system process of smart grid [50]. The data transmission in the smart grid has a great role, if transmitted data hacked by any hacker it can destroy the operation of whole power system by distortion in real time meter readings, Billing status that how many units consumed by consumer, Wrong information at operator centre regarding different equipments connected by transmission, distribution cyber security required [51].

\subsection{Interoperability}

Different devices have different sets of rules for operation, interoperability has a great role for the future smart Distribution system, and it also comes under one of the challenges of the smart grid. Incorporation of IoT with different devices and protocols are input challenge for IoT's changeover to as mart grid [28].

\section{CONCLUSION}

The upgrade of the smart grid highly depends upon consistency and protection against sudden fault, efficiency, real time functioning and robustness with high data rate and low latency capability. In this paper, Opportunities and challenges in the deployment of the IoT has discussed, a low power wide area network used in the smart grid as a communication infrastructure as an enabling technology. We have presented the modern application of IoT and discuss their features to achieve high speed, low latency, low-power communication and low deployment cost for a large number of devices. Finally, we have outlined the opportunities and challenges in realizing the IoT as the smart choice for smart grid.

\section{FUTURE WORK}

To make Smart grid more reliable and efficient, advancement in communication infrastructure is requires. Deployment of internet of things has significance role, best solutions for the researcher to analyse problems associated with serious losses in transmission and distribution line, effective utilization of renewable energy resources with storage, smart charging of V2G, power theft detection, load forecasting, grid optimization and real time smart energy management at consumers end to develop the efficient use of electricity; enhance the level of ease and customer satisfaction.

\section{REFERENCES}

[1] K. M. R. Eswar, "Smart Grid-Future for Electrical Systems," International Journal of Electrical and Electronics Research, vol. 3, no. 2, pp. 603-612, June 2015.

[2] H. H. Alhelou, M. E. H.-Golshan, T. C. Njenda and P. Siano, "A Survey on Power System Blackout and Cascading Events: Research Motivations and Challenges," Energies, vol. 12, no. 4, pp.682-710, Feb 2019, doi: 10.3390/en12040682.

[3] M. A. Elgenedy, A. M. Massoud and S. Ahmed, "Smart grid self-healing: Functions, applications, and developments," 2015 First Workshop on Smart Grid and Renewable Energy (SGRE), 2015, pp. 1-6, doi: 10.1109/SGRE.2015.7208737.

[4] V. C. Gungor, B. Lu and G. P. Hancke, "Opportunities and Challenges of Wireless Sensor Networks in Smart Grid," in IEEE Transactions on Industrial Electronics, vol. 57, no. 10, pp. 3557-3564, Oct. 2010, doi: 10.1109/TIE.2009.2039455

[5] A. H. A.-Badi, R. Ahshan, N. Hosseinzadeh, R. Ghorbani and E. Hossain, "Survey of Smart Grid Concepts and Technological Demonstrations Worldwide Emphasizing on the Oman Perspective," Applied System Innovation, vol. 3, no. 5, Jan 2020, doi:10.3390/asi3010005.

[6] A. Ghasempour, "Internet of Things in Smart Grid: Architecture, Applications, Services, Key Technologies, and Challenges," Inventions, vol. 4, no. 1, p. 22, March 2019, doi: 10.3390/inventions4010022.

[7] J. Zheng, D. W. Gao and L. Lin, "Smart Meters in Smart Grid: An Overview," 2013 IEEE Green Technologies Conference (GreenTech), 2013, pp. 57-64, doi: 10.1109/GreenTech.2013.17.

[8] A. Hirsch, Y. Parag and J. Guerrero, "Micro grids: A review of technologies, key drivers, and outstanding issues," Renewable and Sustainable Energy Reviews, vol. 90, pp. 402-411, 2018, doi: 10.1016/j.rser.2018.03.040.

[9] P. Zand, S. Chatterjea, K. Das and P. Havinga, "Wireless Industrial Monitoring and Control Networks: The Journey So Far and the Road Ahead," Journal of Sensor and Actuator Networks, vol. 1, no. 2, pp. 123-152, Jan 2012, doi: $10.3390 /$ jsan1020123. 
[10] O. Vermesan and P. Friess, "Internet of Things: Converging Technologies for Smart Environments and Integrated Ecosystems," River Publishers Series in Communication River, Denmark: Aalborg, 2013.

[11] S. S. Ali and B. J. Choi, "State-of-the-Art Artificial Intelligence Techniques for Distributed Smart Grids: A Review," Electronics, vol. 9, no. 6, pp. 1030, June 2020, doi: 10.3390/electronics9061030.

[12] V. C. Gungor et al., "Smart Grid Technologies: Communication Technologies and Standards," in IEEE Transactions on Industrial Informatics, vol. 7, no. 4, pp. 529-539, Nov. 2011, doi: 10.1109/TII.2011.2166794.

[13] S. Galli, A. Scaglione and Z. Wang, "Power Line Communications and the Smart Grid," 2010 First IEEE International Conference on Smart Grid Communications, 2010, pp. 303-308, doi: 10.1109/SMARTGRID.2010.5622060.

[14] R. Ma, H. Chen, Y. Huang and W. Meng, "Smart Grid Communication: Its Challenges and Opportunities," in IEEE Transactions on Smart Grid, vol. 4, no. 1, pp. 36-46, March 2013, doi: 10.1109/TSG.2012.2225851.

[15] H. Li, L. Lai and W. Zhang, "Communication Requirement for Reliable and Secure State Estimation and Control in Smart Grid," in IEEE Transactions on Smart Grid, vol. 2, no. 3, pp. 476-486, Sept. 2011, doi: 10.1109/TSG.2011.2159817.

[16] W. Wang, Y. Xu, and M. Khanna: "A survey on the communication architectures in smart grid," Computer network, vol. 55, no. 15, pp. 3604-3629, 2011, doi: 10.1016/j.comnet.2011.07.010.

[17] A. Bensky, "Wireless Personal Area Networks," Short Range Wireless Communication (Third Edition), Ch. 2, pp. 317-360, USA: Sciencedirect, Aug 2019.

[18] K. Sayed and H. A. Gabbar, "SCADA and smart energy grid control automation," Smart Energy Grid Engineering, pp. 481-514, USA: Academic Press, Jan 2017.

[19] M. Kuzlu, M. Pipattanasomporn, S. Rahman, "Communication network requirements for major smart grid applications in HAN,NAN and WAN," Computer Networks, vol. 67, pp. 74-88, 2014, doi: 10.1016/j.comnet.2014.03.029.

[20] M. F. Khan, A. Jain, V. Arunachalam and A. Paventhan, "Communication technologies for smart metering infrastructure," 2014 IEEE Students' Conference on Electrical, Electronics and Computer Science, 2014, pp. 1-5, doi: 10.1109/SCEECS.2014.6804427.

[21] C. Bekara, "Security Issues and Challenges for the IoT-based Smart Grid," Procedia Computer Science, vol. 34, pp. 532-537, 2014, doi: 10.1016/j.procs.2014.07.064.

[22] A. Kailas, V. Cecchi, and A. Mukherjee, "A Survey of Communications and Networking Technologies for Energy Management in Buildings and Home Automation," Journal of Computer Networks and Communications, vol. 2012, 2012, doi: 10.1155/2012/932181.

[23] Y. Li, X. Cheng, Y. Cao, D. Wang and L. Yang, "Smart Choice for the Smart Grid: Narrowband Internet of Things (NB-IoT)," in IEEE Internet of Things Journal, vol. 5, no. 3, pp. 1505-1515, June 2018, doi: 10.1109/JIOT.2017.2781251.

[24] X. Fang, S. Misra, G. Xue and D. Yang, "Smart Grid-The New and Improved Power Grid: A Survey," in IEEE Communications Surveys \& Tutorials, vol. 14, no. 4, pp. 944-980, Fourth Quarter 2012, doi: 10.1109/SURV.2011.101911.00087.

[25] M. S. Hadi, A. Q. Lawey, T. E. H. E.-Gorashi and J. M. H. Elmirghani, "Big data analytics for wireless and wired network design: A survey,” Computer Networks, vol. 132, pp. 180-199, Jan 2018, doi: 10.1016/j.comnet.2018.01.016.

[26] Lars Torsten Berger, Andreas Schwager, and J. Joaquín Escudero-Garzás, "Power Line Communications for Smart Grid Applications," Journal of Electrical and Computer Engineering, Vol. 2013, March 2013, doi: $10.1155 / 2013 / 712376$

[27] D. Yang, Y. Xu and M. Gidlund, "Wireless Coexistence between IEEE 802.11- and IEEE 802.15.4-Based Networks: A Survey," International Journal of Distributed Sensor Networks, vol. 7, no. 1, pp. 912152, 2011, doi: $10.1155 / 2011 / 912152$.

[28] N. H. Motlagh, M. Mohammadrezaei, J. Hunt and B. Zakeri, "Review Internet of Things (IoT) and the Energy Sector," Energies, vol. 13, no. 2, pp. 494, Jan 2020, doi: 10.3390/en13020494.

[29] X. Luo, J. Wang, M. Dooner and J. Clarke, "Overview of current development in electrical energy storage technologies and the application potential in power system operation," Applied Energy, vol. 137, pp. 511-536, Jan. 2015, doi: 10.1016/j.apenergy.2014.09.081.

[30] The Smart Grid: An Introduction. 2018. [Online]. Available: https://www.energy.gov/oe/downloads/smart-gridintroduction-0, Accessed: June 23, 2021.

[31] P. Sethi and S. R. Sarangi, "Internet of Things Architectures, Protocols, and Applications," Journal of Electrical and Computer Engineering, vol. 2017, 2017, doi: 10.1155/2017/9324035.

[32] H. Su, M. Qiu and H. Wang, "Secure wireless communication system for smart grid with rechargeable electric vehicles," in IEEE Communications Magazine, vol. 50, no. 8, pp. 62-68, August 2012, doi: 10.1109/MCOM.2012.6257528.

[33] W. Shu-wen, "Research on the key technologies of IOT applied on Smart Grid," 2011 International Conference on Electronics, Communications and Control (ICECC), 2011, pp. 2809-2812, doi: 10.1109/ICECC.2011.6066418.

[34] Emilio Ancillotti, Raffaele Bruno and Marco Conti," The role of communication systems in smart grids: Architectures, technical solutions and research challenges", Computer communications, Vol.36, pp.1665-1697, December 2013, doi:10.1016/j.comcom.2013.09.004.

[35] R. R. Mohassel, A. Fung, F. Mohammadi and K. Raahemifar, "A survey on Advanced Metering Infrastructure," International Journal of Electrical Power \& Energy Systems, vol. 63, pp. 473-484, July 2014, doi: 10.1016/j.ijepes.2014.06.025. 
[36] E. Hossain, Z. Han and H. V. Poor, "Smart Grid Communications and Networking," New York, NY, USA: Cambridge Univ. Press, 2012.

[37] R. Khan and J. Khan, "A comprehensive review of the application characteristics andtraffic requirements of a smart grid communications network," Computer Networks, vol. 57, no. 3, pp. 825-845, 2013, doi: 10.1016/j.comnet.2012.11.002.

[38] Y. Yan, Y. Qian, H. Sharif and D. Tipper, "A Survey on Smart Grid Communication Infrastructures: Motivations, Requirements and Challenges," in IEEE Communications Surveys \& Tutorials, vol. 15, no. 1, pp. 5-20, First Quarter 2013, doi: 10.1109/SURV.2012.021312.00034.

[39] V. C. Gungor et al., "A Survey on Smart Grid Potential Applications and Communication Requirements," in IEEE Transactions on Industrial Informatics, vol. 9, no. 1, pp. 28-42, Feb. 2013, doi: 10.1109/TII.2012.2218253.

[40] A. Usman and S. Shami, "Evolution of communication technologies for smart gridapplications," Renewable \& Sustainable Energy Reviews, vol. 19, pp. 191-199, 2013, doi: 10.1016/j.rser.2012.11.002.

[41] L. Ardito, G. Procaccianti, G. Menga, and M. Morisio, "Smart grid technologies in Europe: An overview," Energies, vol. 6, no. 1, pp. 251-281, 2013, doi: 10.3390/en6010251.

[42] M. Kuzlu and M. Pipattanasomporn, "Assessment of communication technologies and network requirements for different smart grid applications," 2013 IEEE PES Innovative Smart Grid Technologies Conference (ISGT), 2013, pp. 1-6, doi: 10.1109/ISGT.2013.6497873.

[43] J. Ding, M. Nemati, C. Ranaweera and J. Choi, "IoT Connectivity Technologies and Applications: A Survey," in IEEE Access, vol. 8, pp. 67646-67673, 2020, doi: 10.1109/ACCESS.2020.2985932.

[44] Y. A. Adediran, H. Lasisi and O. B. Okedere, "Interference management techniques in cellular networks: A review," Cogent Engineering, vol. 4, no. 1, April 2017, doi: 10.1080/23311916.2017.1294133.

[45] V. Namboodiri, V. Aravinthan, S. N. Mohapatra, B. Karimi and W. Jewell, "Toward a Secure Wireless-Based Home Area Network for Metering in Smart Grids," in IEEE Systems Journal, vol. 8, no. 2, pp. 509-520, June 2014, doi: 10.1109/JSYST.2013.2260700.

[46] T. Andrysiak, A. Saganowski and P. Kiedrowski, "Research Article Anomaly Detection in Smart Metering Infrastructure with the Use of Time Series Analysis," Journal of Sensors, vol. 2017, July 2017, doi: $10.1155 / 2017 / 8782131$.

[47] C. Wilson, T. Hargreaves and R. H.-Baldwin, "Benefits and Risk of Smart home Technologies," Energy Policy, vol. 103, pp.72-83, Jan 2017, doi: 10.1016/j.enpol.2016.12.047.

[48] T. D. P. Mendes, R. Godina, E. M. G. Rodrigues, J. C. O. Matias and J. P. S. Catalão, "Smart Home Communication Technologies and Applications: Wireless Protocol Assessment for Home Area Network Resources," Energies, vol. 8, pp. 7279-7311, July 2015, doi: 10.3390/en8077279.

[49] C. Kalalas, L. Thrybom and J. Alonso-Zarate, "Cellular Communications for Smart Grid Neighborhood Area Networks: A Survey," in IEEE Access, vol. 4, pp. 1469-1493, 2016, doi: 10.1109/ACCESS.2016.2551978.

[50] M. R. Asghar, G. Dán, D. Miorandi and I. Chlamtac, "Smart Meter Data Privacy: A Survey," in IEEE Communications Surveys \& Tutorials, vol. 19, no. 4, pp. 2820-2835, Fourthquarter 2017, doi: 10.1109/COMST.2017.2720195.

[51] R. Bikmetov, M. Y. A. Raja and T. U. Sane, "Infrastructure and applications of Internet of Things in smart grids: A survey," 2017 North American Power Symposium (NAPS), 2017, pp. 1-6, doi: 10.1109/NAPS.2017.8107283.

\section{BIOGRAPHY OF AUTHORS}

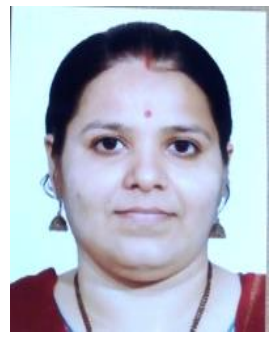

Payal Soni is currently pursuing Ph.D. degree in Electronics and Communications Engineering from SRMIST, SRM University Tamilnadu India. She has worked as teaching faculty in engineering colleges and she is having 9 years of experience in the field of Electrical and Electronics Engineering. Her research interests lie in the areas of Smart Grid communication technologies and Smart energy management systems.

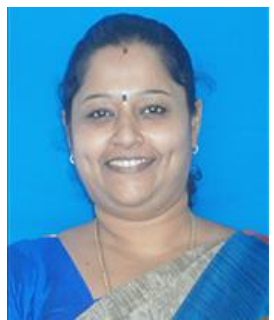

Dr. J. Subhashini is Assistant Professor in Department of Electronics and Communication Engineering, SRMIST, SRM University Tamilnadu India. She has done Ph.D. degree in wireless communication. She is having 20 years of experience in the field of Electronics and Communication Engineering and guided M. Tech and Ph.D. Students. Since 2001She has published more than 30 Peer review Scientific papers in the area of wireless communication. 